\title{
MJFAS MALAYSIIA JOURNAL OF

\section{An effect of physical exercise-induced fatigue on the vital sign parameters: A preliminary study}

\author{
Zulkifli Ahmad a, b, ${ }^{,}$, Mohd Najeb Jamaludin ${ }^{\mathrm{b}}$, Kamaruzaman Soeed ${ }^{\mathrm{b}}$ \\ a Institute of Human Centered Engineering, Universiti Teknologi Malaysia, Skudai 81310, Johor, Malaysia \\ b Human Engineering Group, Faculty of Mechanical Engineering, Universiti Malaysia Pahang, 26600, Pekan, Pahang, Malaysia \\ * Corresponding author: zulkola84@gmail.com
}

\section{Article history}

Received 31 August 2018

Revised 12 October 2018

Accepted 7 November 2018

Published Online 14 April 2019

\section{Graphical abstract}

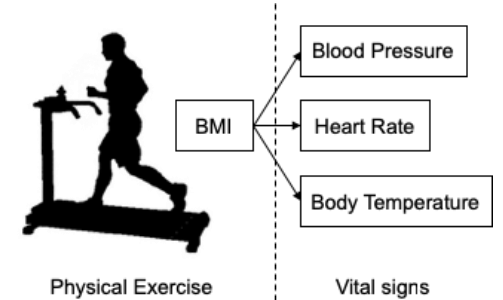

\begin{abstract}
Vital sign monitoring is an important body measurement to identify health condition and diagnose any disease and illness. In sports, physical exercise will contribute to the changes of the physiological systems, specifically for the vital signs. Therefore, the objective of this study was to determine the effect of physical fatigue exercise on the vital sign parameters. This is significant for the fitness identification and prediction of each individual when performing an exercise. Five male subjects with no history of injuries and random BMI were selected from students of biomedical engineering, Universiti Teknologi Malaysia. Based on the relationship between physical movement and physiology, the parameters considered were heart rate, blood pressure, and body temperature. Subjects were required to run on the treadmill at an initial speed of $4 \mathrm{~km} / \mathrm{h}$ with an increase of $1 \mathrm{~km} / \mathrm{h}$ at every 2 minutes interval. The effect of exercise was marked according to the fatigue protocol where the subject was induced to the maximum condition of performance. All parameters were measured twice, for pre and post exercise-induced protocol. The analysis of relationship of each parameter between pre and post fatigue was $p<0.05$. The results revealed that the heart rate and gap between blood pressure's systolic and diastolic were greater for all categories except underweight, where the systolic blood pressure dropped to below $100 \mathrm{mmHg}$ at the end of exercise. Also, the body temperature was slightly declined to balance the thermoregulatory system with sweating. Hence, the vigorous physical movement could contribute to the active physiological system based on body metabolism. Heart rate and blood pressure presented significant effects from the fatiguing exercise whereas the body temperature did not indicate any distinguishable impact. The results presented might act as the basis of reference for physical exercise by monitoring the vital sign parameters.
\end{abstract}

Keywords: Vital signs, blood pressure, heart rate, body temperature, exercise-induced fatigue

\section{INTRODUCTION}

Vital signs are a measurement of the body's most fundamental components in the nursing assessment (Chester \& Rudolph, 2011) There are five vital sign organs, which control our body's physiology, namely the brain, heart, kidneys, liver, and lungs. Four main vital signs that are routinely monitored by medical practitioners and healthcare providers are heart rate, body temperature, blood pressure and respiration rate. There are a number of health issues that treatment benefits from vital sign monitoring (Yilmaz et al., 2010), and is also widely used in emergency departments (Ljunggren et al., 2016) as a first indication for screening the health condition of the patient before any further treatment. It is known to reduce the number of mortality due to unexpected events such as cardiac arrest through recognition of an abnormal sign in the vital locations (Nurmi et al., 2005).

Previous studies have investigated an effect of exercise on two parameters' response such as heart rate (Chen et al., 2015; Itoh et al., 2013; Mazzoleni at al., 2016), blood pressure (Douglas et al., 1987; Itoh et al., 2013), respiratory rate (McEntire et al., 2016), and body temperature (Towey, Easton, Simpson, \& Pedlar, 2016). However, combination of three vital sign parameters in exercise response are still lacking and less studied. Furthermore, the vital signs are not broadly used in sports with the exception of heart rate and respiratory monitoring due to the limitation on the device portability.

Physical fatigue or muscle fatigue is not merely an effect of activities in the muscular system, but it is also reflected in other physiological systems in our body. In order to identify systems that contribute most to physical fatigue, the vital sign parameters can be observed. Nevertheless, the vital organ system is not commonly considered as part of the muscular system. The muscle is linked indirectly to the heart which pumps oxygenated blood throughout the body, including the muscles, in order to simultaneously remove deoxygenated blood. Therefore, any exercise intensity using the muscles will influence the physiological system, and this can be linked by monitoring the vital sign parameters.

\section{Blood pressure}

Blood pressure (BP) is an important vital sign parameter for early detection of cardiovascular disorders and circulatory system diseases in pre-sick patients by focusing on the capability of heart to pump the blood (Lopez et al., 2010). Commonly, exercise testing is conducted to reveal any metabolic problem by observing the pattern of blood pressure, and it also can be associated with the heart rate variability (Itoh et al., 2013). 
For the sedentary population, the peak systolic BP of $214 \mathrm{mmHg}$ and above after exercise can be predicted as the high-risk possibility of hypertension occurrence based on the guideline from American Heart Association (AHA) (Caselli et al., 2016). Therefore, physical activity has a strong agreement to BP as it is influenced by body weight, gender and age (Itoh et al., 2013) whereas the robust determinant for sports disciplines is body mass index (Caselli et al., 2016).

\section{Cardiorespiratory}

The easiest way to determine the body fitness is by referring to the resting heart rate which is measured in the early morning before getting out the bed. However, this variable is an independent risk factor, due to the surrogate marker of subclinical disease states or a marker of poor physical fitness (Jensen et al., 2013). Since there is a close relationship between heartbeat and respiration of body fitness, most of the researchers use the term "cardiorespiratory fitness" to describe the effect of both physiological systems (cardiovascular and respiratory) towards fitness. On the other hand, the cardiorespiratory fitness does not differ by race and Hispanic origin, or by the family income-topoverty ratio (Gahche et al., 2014). Hence, the maximum rate of oxygen consumption $\left(\mathrm{VO}_{2} \max \right)$ is used to express the cardiorespiratory fitness measurement as the maximum oxygen uptake into the body during an exercise test.

In cardiorespiratory, there are two main parameters involved, which are heart rate and breathing rate. These parameters are measured based on the activities from the internal organs heart and lung. For heart rate, the measurement is based on the number of heartbeats performed within one minute while the breathing rate is reflected by the number of breathing cycles within a minute. One breathing cycle consists of a complete inspiration and expiration process. Normal heart rate and breathing rate are ranging between $60-100$ beats per minute (bpm) and 12-20 respiratory rate (rr), respectively. However, for the active person especially athletes with excellent body fitness, these values can fall to a minimum of $40 \mathrm{bpm}$ and $10 \mathrm{rr}$ known as bradycardia. The heart and breathing rate will be lower than normal because heart is in an efficient condition to circulate the blood around the body with less force required.

\section{Body temperature}

The human body can sustain a constant body temperature at $37^{\circ} \mathrm{C}$ as a result of a balance between heat production and heat dissipation by the thermoregulatory system (Tanda, 2016). However, during an exercise, body heat is generated based on the activities and movements performed (Neves et al., 2016). This is due to the muscle contraction that requires oxygen as a fuel to be activated (similar to an engine) in order for the process to become faster which produces heat depending on the metabolism rate.

Athletes are particularly susceptible to heat-related illnesses because they have frequent training every day under high ambient temperature. Under this condition, the rate of heat production exceeds the heat dissipation and the core temperature tends to increase. Hence, a "cooling mechanism" will be activated to counter the heat production by increasing the sweating and evaporation concurrently. On the other hand, if elevated body temperature occurs in extreme conditions, which is more than $40^{\circ} \mathrm{C}$, it will become life-threatening and fatal. Furthermore, it can result in the enormous damage to the human body and performance degradation (Adams et al., 2016) which will lead to collapse owing to hyperthermia (Tanda, 2016).

To date, studies on exercise effects are focused on specific parameter of the vital signs such as blood pressure, heart rate, and temperature individually (Cruz, 2016; Gläser et al., 2013; Tuka et al., 2015; Weigert et al., 2018). Thus far, there is an insufficient study in correlating the vital sign parameters concurrently with intense exercise to the level of fatigue in order to reveal the extreme conditions of the body physiology. Therefore, this study was aimed to investigate the significant vital sign parameters which can be set as a threshold value for fatigue states. These acquired data and analysis are believed to provide more reliable indicators that are required in physical fatigueinduced exercise.

\section{METHODOLOGY}

\section{Subjects}

All five male volunteeres were randomly selected from different backgrounds to participate in this study. The subjects' demography (mean \pm SD age: $24.2 \pm 1.6$ years, height: $170.3 \pm 4.74 \mathrm{~cm}$, weight: $68.1 \pm 12.83 \mathrm{~kg}$ ) with no previous history of injuries and accidents. The body mass index (BMI) was calculated in the $\mathrm{kg} / \mathrm{m} 2$ unit. Inclusion criterion of the subject was sedentary people with no heart disease problem and passing the screening procedure. The study was conducted in a safe and controlled environment in the laboratory without any possibility for injury to occur. All subjects were required to complete and sign a writted informed consent form prior to testing.

\section{Screening procedure}

Prior to any warm-up activity, the subject body was attached to the 12-lead electrocardiogram (ECG) device to monitor the heart condition in the resting position. All ten gel-type $\mathrm{Ag} / \mathrm{AgCl}$ wet-electrodes were placed on the body based on the American Heart Association (AHA) protocol. It was recommended by many sporting organisations for conducting the pre-participating screening procedure before attending any competitive events, especially for a young athlete (Asif \& Prutkin, 2015). PC-based resting ECG (Welch Allyn, United States) was used to perform the screening procedure for both conditions at resting and exercise on the treadmill. It was necessary to identify any heart abnormality in each subject before performing the fatigue experiment.

However, in vital sign monitoring, heart rate was considered enough to identify the exercise zone condition. Furthermore, peripheral oxygen saturation $(\mathrm{SpO} 2)$ was important to be taken into account for identifying the amount of oxygen in the blood. More precisely, it was the percentage of oxygenated haemoglobin $\left(\mathrm{HbO}_{2}\right)$ divided into the total of haemoglobin $(\mathrm{Hb}+\mathrm{HbO} 2)$ as presented in Eq. 1. For a normal $\mathrm{SpO}_{2}$, values varied between 95 and $100 \%$. This value was one of the inclusion criteria for subject selection in a fatigue protocol.

$$
\mathrm{SpO}_{2}=\frac{\mathrm{HbO}_{2}}{\mathrm{Hb}+\mathrm{HbO}_{2}}
$$

In order to calculate the percentage of the exercise zone condition based on the heart rate, it could be derived from Karvonen's formula in Eq. 2 below:

$$
\begin{array}{r}
H R_{\max }=220-A g e=188 \mathrm{bpm} \\
H R_{\text {rest }}=60 \mathrm{bpm}, H R_{\text {ex }}=160 \mathrm{bpm} \\
\% H R=\frac{\left(H R_{\text {ex }}-H R_{\text {rest }}\right)}{\left(H R_{\max }-H R_{\text {rest }}\right)} \times 100 \\
\% H R=\frac{(160-60) \mathrm{bpm}}{(188-60) \mathrm{bpm}} \times 100=85 \%
\end{array}
$$

HRmax and HRrest are maximum heart rate and resting heart rate, respectively. Interpolation between these two parameters contributed to the level of exercise in percentage value. To measure both parameters (heart rate and $\mathrm{SpO} 2$ ), hence, the commercial pulse oxymetry device was selected to use in this screening procedure.

\section{Exercise-induced fatigue protocol}

This fatigue-induced protocol was conducted following the method proposed by Ahmad et al. (2018). Nevertheless, the equipment used in this experiment was changed to the vital spot monitor (Welch Allyn, United States). The advantage of this equipment was an integrated vital sign monitoring particularly for blood pressure, heart rate and body temperature as shown in Fig. 1. A control lab environment was set up to ensure the temperature, ambience, and humidity were similar in every experiment conducted.

The subjects were educated with the experiment protocol by referring to the consent form provided and signed for declaration. These selected subjects were those who passed the screening procedure. Vital sign parameters (blood pressure, heart rate and body temperature) were measured to be stored as resting data. The subjects were required to run 
on the treadmill with an initial speed of $4 \mathrm{~km} / \mathrm{h}$ and the intensity was increased by $1 \mathrm{~km} / \mathrm{h}$ at every 2 minutes interval. During running, the subjects would identify their fatigue condition based on the Borg's scale rating. The scale has 10-points starting with 1 (light) and ending with 10 (fatigue). In order to stop an experiment, the subjects are required to stop the treadmill by themselves when the condition of volitional fatigue was achieved, ensuring the subjects do not force themselves which could lead to injury. The vital sign parameters were recorded again and kept as post-fatigue data. The experiment was conducted with the necessary medical aid prepared for any unexpected situation. Finally, the subjects performed their own recovery method to ensure their condition went back to normal.

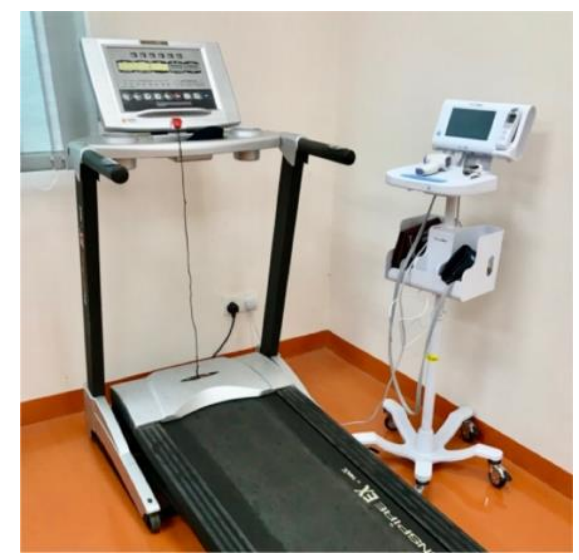

Fig. 1 Equipment setup with vital sign monitor and treadmill.

\section{RESULTS AND DISCUSSION}

The findings of the present study suggested that the heart rate (HR) was consistent with findings from Neves et al., (2016) that showed a significant increase in HR during exercise (Fig. 2). It was followed by steady increase in the blood oxygen saturation $\left(\mathrm{SpO}_{2}\right)$ as time went on. However, these results were based on data of 5 minutes and it clearly remained in the steady state of $\mathrm{HR}$ afterwards as proved by Bellenger et al., (2016). Although both parameters $\mathrm{HR}$ and $\mathrm{SpO}_{2}$ were measured differently, they came from one source of reference, which was peripheral blood volume. Therefore, it has a close relationship with the characteristics of pattern and trend. When an exercise was performed, the heart would pump aggressively to supply adequate oxygen to the muscle for contraction and $\mathrm{SpO}_{2}$ or oxygen capacity in each volume was presented with the capability of haemoglobin to transport oxygen. Besides that, this oxygen was related to the volume of oxyhemoglobin compared to the deoxyhemoglobin in the arterial blood vessels (Liang et al., 2016). On the other hand, the fitness of the subjects could be associated with the $\mathrm{SpO}_{2}$ and $\mathrm{HR}$.

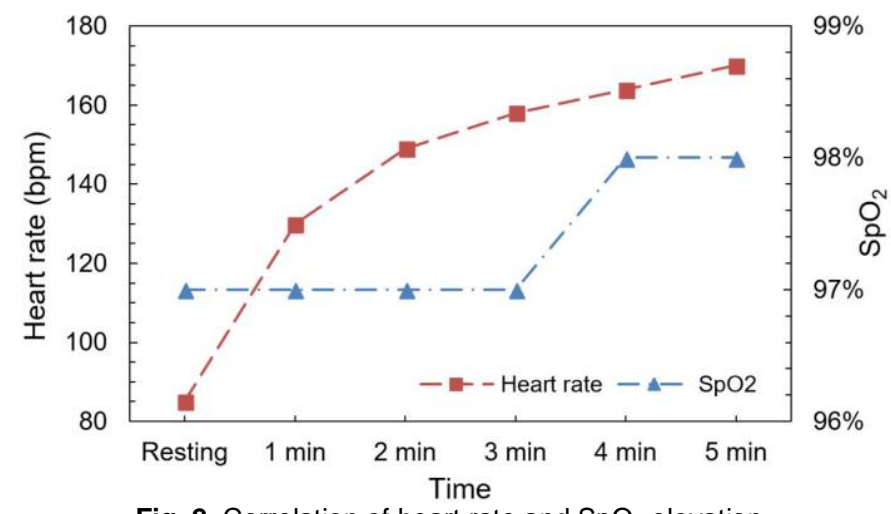

Fig. 2 Correlation of heart rate and $\mathrm{SpO}_{2}$ elevation.

By comparing the measurement of heart rate by using oxymetry and ECG in Fig. 3, it was shown that ECG had more detailed evidence regarding heart characteristics for depolarisation and repolarisation of the ventricles. The pattern of ECG presented information that could be used to identify and diagnose any heart-related disease. Fig. 3 depicted the ECG for lead I, II and III that represented the heartbeat in different angles of measurement based on the location of electrodes to comply with Einthoven's Law. The peak of ECG was denoted as R and the distance between peaks was represented for the heart rate variability (HRV) which was calculated from the number of boxes with value of $25 \mathrm{~mm} / \mathrm{sec}$ in the ECG paper. By observing these ECG signals, Fig. 3(a) and Fig. 3(b) indicated the measurement before and after the exercise. The average parameter for heart rate increased from $86 \mathrm{bpm}$ to $111 \mathrm{bpm}$, while the distance of overall PQRST was shorter due to the HRV that became faster. For pre and post exercise, the parameters of PQ and QT were decreased from $148 \mathrm{~ms}$ to $135 \mathrm{~ms}$ and $349 \mathrm{~ms}$ to 322 $\mathrm{ms}$, respectively. However, QRS showed the most consistent value from $79 \mathrm{~ms}$ to $80 \mathrm{~ms}$.

In sports application, detailed information of ECG was not a required measurement for heart rate monitoring since the information from the oxymeter was adequate for analysis. Most of the measurement by ECG was used for detecting disease related to the heart problem from its signal waves such as sleep apnea (Sharma \& Sharma, 2016), hypertrophic cardiomyopathy (Grazioli et al., 2015), and arrhythmias (Petrenas et al., 2015). Moreover, the 10-electrodes attached to the body were wired, which disrupting the athlete's movement during exercise and hence, the signal of ECG was difficult to be analysed due to motion artefact. In contrast, there was previous researcher that used ECG as a measurement for heart rate, but it was limited to the singlelead with minimum two electrodes (Orchard et al., 2013; Sharma \& Sharma, 2016) compared to these 12-leads. Also, the ECG measurement by using PC based resting ECG was constrained for only 10 seconds and not suited for the real-time monitoring condition. Hence, the simplest way to measure heart rate could be done by using oxymeter rather than ECG.
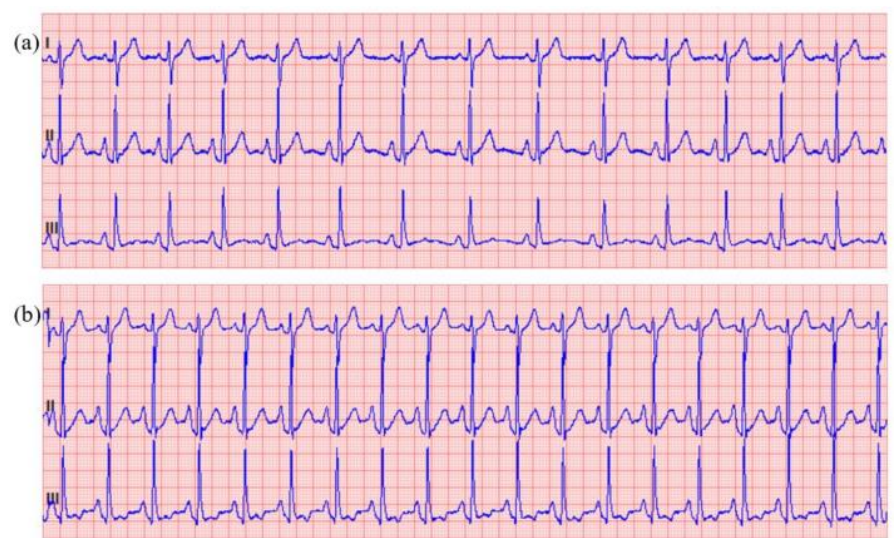

Fig. 3 ECG rhythm of lead I, II and III for (a) before and (b) after exercise.

Table 1 shows the details of vital signs selected for resting and postfatigue experiments in a different type of BMI categories. There were four categories to be considered in this study to represent the wholebody composition category: underweight $\mathrm{BMI}<18.5$; healthy $\mathrm{BMI}$ 18.5-25; overweight BMI 25-30 and obese $\mathrm{BMI}>30$. Then this data was divided the blood pressure into systolic and diastolic pressure for upper and lower threshold. Hence, Fig. 4 exhibits the changes in blood pressure for systolic and diastolic pressure in pre and post exercises by subject assessment. Strong evidence of blood pressure was found to be increased in post-exercise for all categories whereas contrast for the underweight category. It was declined from $114 \mathrm{mmHg}$ to $99 \mathrm{mmHg}$ and $78 \mathrm{mmHg}$ to $50 \mathrm{mmHg}$ for both systolic and diastolic pressure, respectively. On the other hand, diastolic pressure was deteriorated in all categories except for subject 2, which was BMI overweight. The difference in diastolic between pre and post exercise was slightly reduced 1 or $2 \mathrm{mmHg}$ for the healthy group while the other group would contribute the changes up to $49 \mathrm{mmHg}$.

The most surprising aspect of the data was in the gap between systolic and diastolic pressure when it developed the bigger size in postexercise condition regardless BMI category. The gap size for preexercise was consistent from the beginning by $40 \pm 4.43 \mathrm{mmHg}$, and it 
was grown up to $57 \pm 6.8 \mathrm{mmHg}$ for post exercise. Unfortunately, the measurement of blood pressure by using this device has somedrawbacks regarding data effectiveness. At the end of the experiment, the pressure was dropped due to body recovery, and it was difficult to detect the constant pressure once it was finished.

Table 1 Demographic data of subjects after fatigue exercise.

\begin{tabular}{|c|c|c|c|c|c|c|c|c|c|c|}
\hline \multicolumn{11}{|c|}{ Blood Pressure $(\mathrm{mmHg})$} \\
\hline Subject & BMI & Category & & Systolic & Diastolic & Gap & $\mathrm{HR}^{\alpha}(\mathrm{bpm})$ & $\mathrm{BT}^{\beta}\left({ }^{\circ} \mathrm{C}\right)$ & HR (max) & Time (s) \\
\hline \multirow{2}{*}{1} & \multirow{2}{*}{32.8} & \multirow{2}{*}{ Obese } & Pre & 125 & 81 & 44 & 66 & 36.8 & \multirow{2}{*}{155} & \multirow{2}{*}{715} \\
\hline & & & Post & 132 & 68 & 65 & 131 & 36.6 & & \\
\hline \multirow{2}{*}{2} & \multirow{2}{*}{25.3} & \multirow{2}{*}{ Overweight } & Pre & 111 & 66 & 45 & 69 & 36.7 & \multirow{2}{*}{196} & \multirow{2}{*}{775} \\
\hline & & & Post & 126 & 73 & 53 & 119 & 36.9 & & \\
\hline \multirow{2}{*}{3} & \multirow{2}{*}{17.5} & \multirow{2}{*}{ Underweight } & Pre & 114 & 78 & 36 & 95 & 36.7 & \multirow{2}{*}{204} & \multirow{2}{*}{855} \\
\hline & & & Post & 99 & 50 & 49 & 150 & 36.2 & & \\
\hline \multirow{2}{*}{4} & \multirow{2}{*}{20.0} & \multirow{2}{*}{ Healthy } & Pre & 112 & 76 & 35 & 76 & 36.8 & \multirow{2}{*}{191} & \multirow{2}{*}{912} \\
\hline & & & Post & 126 & 73 & 53 & 127 & 36.5 & & \\
\hline \multirow{2}{*}{5} & \multirow{2}{*}{23.0} & \multirow{2}{*}{ Healthy } & Pre & 111 & 72 & 40 & 98 & 36.7 & \multirow{2}{*}{168} & \multirow{2}{*}{700} \\
\hline & & & Post & 134 & 71 & 63 & 145 & 36.3 & & \\
\hline
\end{tabular}

${ }^{\alpha} \mathrm{HR}$ is a heart rate measured in beat per minute

${ }^{\beta}$ BT is body temperature in degree celsius

Since the heart rate measurement represented the point when blood pressure was detected, therefore, this situation was occurred to the heart rate for post exercise as well. The time-delayed caused the body to recover back to the normal by reducing the blood pressure, similarly to the heart rate. However, the data showed sufficient information about the changes in blood pressure when performing an exercise. Moreover, a positive relationship between BMI and blood pressure was excluded for underweight category. Interestingly, underweight was observed to use more acid lactic rather than oxygen from the blood to contract due to fewer numbers of muscle fibre that activated during exercise. Increasing production of lactic acid would cause an unbalanced biochemical reaction in the physiological system. Hence, the underweight category would feel symptoms such as dizziness, faintness and collapse. In this case, the safety aid box has been prepared if such an emergency situation was occurred.

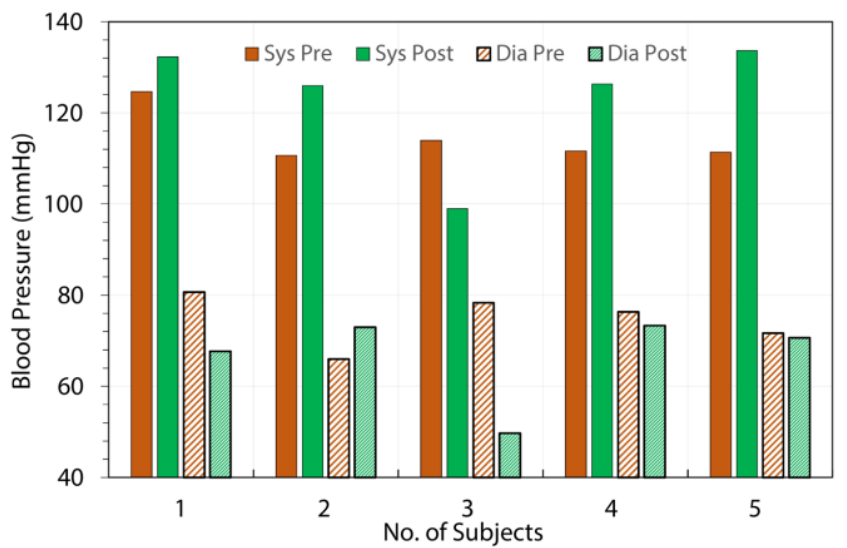

Fig. 4 Effect of blood pressure for pre and post exercise.

There has a strong, positive relationship between heart rate and exercise condition. It was presented in the Fig. 5 when the heart rate was increased dramatically from pre to post exercise. However, the maximum heart rate would indicate the fitness of the subject to perform the experiment based on the time taken of an experiment conducted. The differences in heart rate for each subject could be related to the $\mathrm{BMI}$ as revealed by the value of slope in every linear graph. The lowest degree of slope and the longest time taken were 5.59 degree and 912 seconds, respectively for Subject 4, declaring as an excellent fitness compared to the others. It was followed by Subject 2 and Subject 3, after considering the pre-exercise heart rate. Even though Subject 5 began his pre-exercise heart rate as the highest value, the slope was in the gap of average, which was 6.71. Finally, Subject 1 was indicated as the lowest fitness since the BMI was in the obese category and the highest slope contribution was 9.09 .

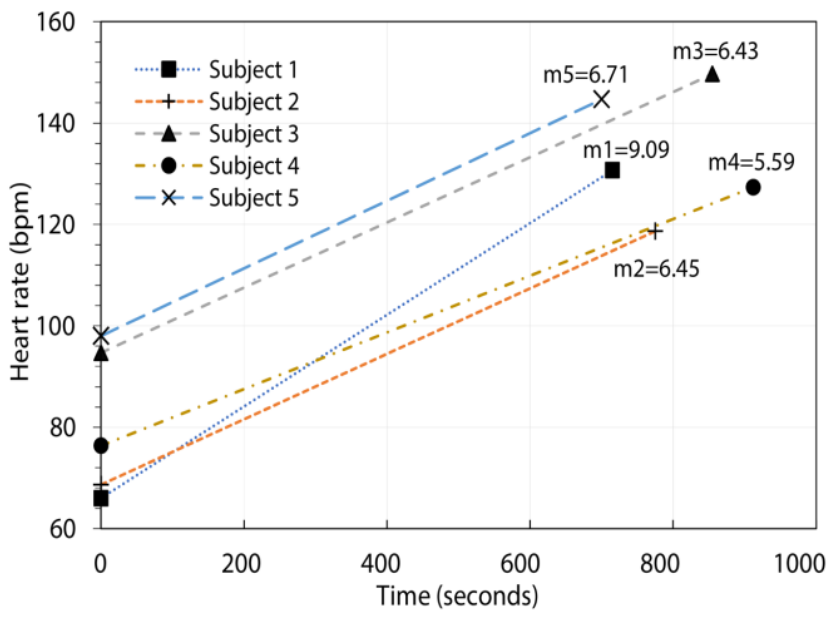

Fig. 5 Heart rate changes due to exercise.

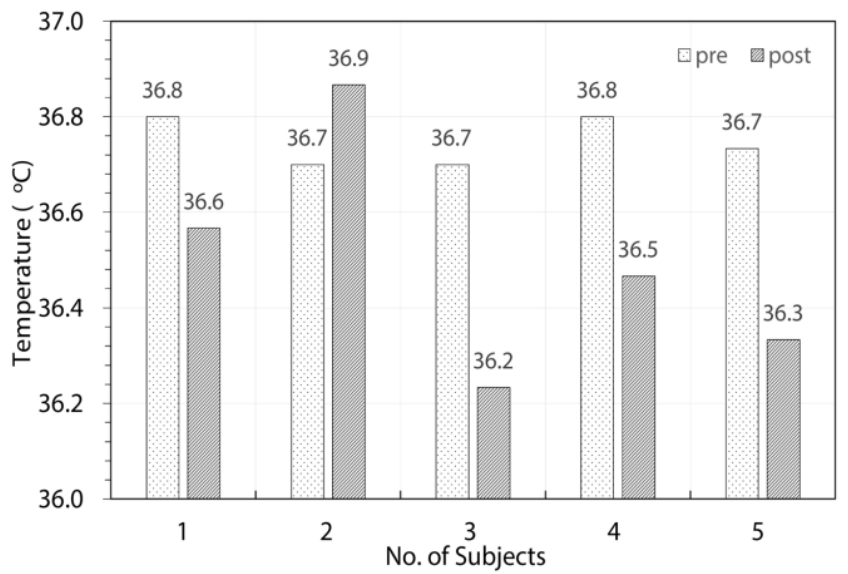

Fig. 6 Body temperature measured for pre and post exercise. 
By referring to the Table 1, the maximum heart rate that could be recorded during an exercise was 155bpm, 196bpm, 204bpm, $191 \mathrm{bpm}$ and $168 \mathrm{bpm}$ according to the subject sequence. Based on the theory of Karvonen's formula in Eq.1, the maximum heart rate of all subjects was ranged from 195.8 \pm 1.64 . However, only Subject 2 and Subject 4 reached the targets while Subject 3 was over, and Subject 1 and 5 were under target. Comparing to both values, the percentage intensities were as following: $67 \%, 100 \%, 104 \%, 96 \%$ and $78 \%$. It was revealed that Subject 1 and 5 did not push their effort to the real fatigue limit based on the heart rate indicator. Conversely, blood pressures for both subjects in post-fatigue exercise showed the highest gap in between systolic and diastolic pressures, which were 65 and $63 \mathrm{mmHg}$. Therefore, the physical fatigue could not be identified merely by one parameter, but another variable should be considered into account to observe the phenomenon in a complete view. Besides, the subjects would not adapt with the fast pacing on the treadmill due to increasing of speed intensity from time to time. This condition would force the subjects to run faster from their comfort pacing and would affect their leg muscles to remain in aerobic exercise. The body temperature of each subject for pre and post exercise was illustrated in Fig. 6. Body temperatures for all subjects were appeared to be in decrease trend, except for the Subject 2. The temperature change was ranged in between $0.32 \pm 0.12^{\circ} \mathrm{C}$. The highest and lowest reductions were observed as $0.5^{\circ} \mathrm{C}$ and $0.2^{\circ} \mathrm{C}$ for Subject 3 and Subject 1 , respectively. Similar result was found from Neves et al. (2016) that the skin temperature was decreased on the active muscles during exercise and the first minutes of the recovery period. In addition, according to Tanda (2016), the duration and exercise intensity would contribute to the heat accumulation, however, the system immediately would be balanced by heat dissipation sufficiently. Since an experiment was conducted indoor and at low ambient temperature, the rate of heat loss was exceeded the heat production and body temperature was tended to decrease.

\section{CONCLUSION}

This study showed that there was a potential to identify the effect of physical exercise-induced fatigue by employing the vital sign parameters. This study indicated parameters that were significant for the fatiguing exercise were blood pressure and heart rate, while the body temperature did not indicate any distinguishable impact. Different BMI could affect the blood pressure after performing exercise-induced fatigue experiment. Besides, the difference gap between systolic and diastolic blood pressure was informative to indicate the fatigue condition. Further study might be conducted on more participants and better insights might be revealed further.

\section{ACKNOWLEDGEMENT}

This work was supported by the Universiti Teknologi Malaysia under the Research University Grant, Tier 2 (Q.J130000.2645.13J73) and also for Universiti Malaysia Pahang and Ministry of HigherEducation Malaysia.

\section{REFERENCES}

Adams, W. M., Hosokawa, Y., Adams, E. L., Belval, L. N., Huggins, R. A., \& Casa, D. J. (2016). Reduction in body temperature using hand cooling versus passive rest after exercise in the heat. Journal of Science and Medicine in Sport, 19(13), 936-940.

Ahmad, Z., Jamaludin, M. N., \& Omar, A. H. (2018). Development of wearable electromyogram for the physical fatigue detection during aerobic activity. Movement, Health \& Exercise, 7(1), 15-25.

Asif, I. M., \& Prutkin, J. M. (2015). Modern standards of ECG interpretation in young athletes: Yield and effectiveness. Journal of Electrocardiology, 48(3), 292-297.

Bellenger, C. R., Thomson, R. L., Howe, P. R. C., Karavirta, L., \& Buckley, J. D. (2016). Monitoring athletic training status using the maximal rate of heart rate increase. Journal of Science and Medicine in Sport, 19(1), 590595.

Caselli, S., Vaquer Segui, A., Quattrini, F., Di Gacinto, B., Milan, A., Assorgi, R., Pelliccia, A. (2016). Upper normal values of blood pressure response to exercise in Olympic athletes. American Heart Journal, 177, 120-128.

Chen, S.-W., Liaw, J.-W., Chang, Y.-J., Chuang, L.-L., \& Chien, C.-T. (2015).
Combined heart rate variability and dynamic measures for quantitatively characterizing the cardiac stress status during cycling exercise. Computers in Biology and Medicine, 63, 133-142.

Chester, J. G., \& Rudolph, J. L. (2011). Vital signs in older patients: Age-related changes. Journal of the American Medical Directors Association, 12(5), 337-343.

Cruz, A. B. (2016). Abnormal blood pressure response to exercise in badminton athletes. Science and Sports, 31(6), 342-346.

Douglas, P. S., O’Toole, M. L., Hiller, W. D. B., Hackney, K., \& Reichek, N. (1987). Cardiac fatigue after prolonged exercise. Circulation, 76(6), 12061213.

Gahche, J., Fakhouri, T., Carroll, D. D., Burt, V. L., Wang, C., \& Fulton, J. E. (2014). Cardiorespiratory fitness levels among U. S. youth aged $12-15$ years : United States, $1999-2004$ and 2012. NCHS Data Brief., 153, 1-8.

Gläser, S., Friedrich, N., Koch, B., Schäper, C., Völzke, H., Felix, S. B., Dörr, M. (2013). Exercise blood pressure and heart rate reference values. Heart Lung and Circulation, 22(8), 661-667.

Grazioli, G., Usin, D., Trucco, E., Sanz, M., Vidal, B., Montserrat, S., Sitges, M. (2015). Differentiating hypertrophic cardiomyopathy from physiological left ventricular hypertrophy: An echocardiographic and ECG approach. European Journal of Preventive Cardiology, May 2015 2, S197.

Itoh, H., Ajisaka, R., Koike, A., Makita, S., Omiya, K., Kato, Y., ... Taniguchi, K. (2013). Heart rate and blood pressure response to ramp exercise and exercise capacity in relation to age, gender, and mode of exercise in a healthy population. Journal of Cardiology, 61(1), 71-78.

Jensen, M. T., Suadicani, P., Hein, H. O., \& Gyntelberg, F. (2013). Elevated resting heart rate, physical fitness and all-cause mortality: A 16-year followup in the Copenhagen Male Study. Heart, 99(12), 882-887.

Liang, T., Yuan, Y. J., \& Member, S. (2016). Wearable medical monitoring systems based on wireless networks: A review. IEEE Sensors, 16(23), 8186-8199.

Ljunggren, M., Castrén, M., Nordberg, M., \& Kurland, L. (2016). The association between vital signs and mortality in a retrospective cohort study of an unselected emergency department population. Scandinavian Journal of Trauma, Resuscitation and Emergency Medicine, 24(21), 1-11.

Lopez, G., Shuzo, M., Ushida, H., Hidaka, K., Yanagimoto, S., Imai, Y., ... Yamada, I. (2010). Continuous blood pressure monitoring in daily life. Journal of Advanced Mechanical Design, Systems, and Manufacturing, 4(1), 179-186.

Mazzoleni, M. J., Battaglini, C. L., Martin, K. J., Coffman, E. M., \& Mann, B. P. (2016). Modeling and predicting heart rate dynamics across a broad range of transient exercise intensities during cycling. Sports Engineering, 19(2), $117-127$.

McEntire, S. J., Smith, J. R., Ferguson, C. S., Brown, K. R., Kurti, S. P., \& Harms, C. A. (2016). The effect of exercise training with an additional inspiratory load on inspiratory muscle fatigue and time-trial performance. Respiratory Physiology and Neurobiology, 230, 54-59.

Neves, E. B., Cunha, R. M., Rosa, C., Antunes, N. S., Felisberto, I. M. V., Vilaça-Alves, J., \& Reis, V. M. (2016). Correlation between skin temperature and heart rate during exercise and recovery, and the influence of body position in these variables in untrained women. Infrared Physics and Technology, 75, 70-76.

Nurmi, J., Harjola, V., Nolan, J., \& Castren, M. (2005). Observations and warning signs prior to cardiac arrest. Should a medical emergency team intervene earlier? Acta Anaesthesiol Scandinavica, 49(5), 702-706.

Orchard, J., Semsarian, C., Freedman, B., \& Neubeck, L. (2013). The AliveCor handheld heart monitor: Turning your iPhone into a single-lead electrocardiogram (ECG). Journal of Science and Medicine in Sport, 16, e61.

Petrènas, A., Marozas, V., Jaruševičius, G., \& Sörnmo, L. (2015). A modified Lewis ECG lead system for ambulatory monitoring of atrial arrhythmias. Journal of Electrocardiology, 48(2), 157-163.

Sharma, H., \& Sharma, K. K. (2016). An algorithm for sleep apnea detection from single-lead ECG using Hermite basis functions. Computers in Biology and Medicine, 77, 116-124.

Tanda, G. (2016). Skin temperature measurements by infrared thermography during running exercise. Experimental Thermal and Fluid Science, 71, 103113.

Towey, C., Easton, C., Simpson, R., \& Pedlar, C. (2016). Conventional and novel body temperature measurement during rest and exercise induced hyperthermia. Journal of Thermal Biology, 63, 124-130.

Tuka, V., Rosa, J., Dědinová, M., \& Matoulek, M. (2015). The determinants of blood pressure response to exercise. Cor et Vasa, 57(3), e163-e167.

Weigert, M., Nitzsche, N., Kunert, F., Lösch, C., \& Schulz, H. (2018). The influence of body composition on exercise-associated skin temperature changes after resistance training. Journal of Thermal Biology, 75(June), $112-119$.

Yilmaz, T., Foster, R., \& Hao, Y. (2010). Detecting vital signs with wearable wireless sensors. Sensors, 10(12), 10837-10862. 